\title{
Substituierte 2-Bromacetessigsäure-Amide als Chelatbildner
}

\author{
Substituted 2-bromacetoacetamides as chelating reagents \\ A. Kettrup und J. Abshagen \\ Lehrstuhl für Anorganische Chemie I der Ruhr-Universität Bochum \\ (Z. Naturforsch. 25 b, $1382-1385$ [1970]; eingegangen am 17. August 1970)
}

\begin{abstract}
The preparation of 2-bromacetoacetamides and their copper chelates is described. The stability constants of these compounds were determined by potentiometric $\mathrm{pH}$-measurements. The change of stability constants by the substituents of the amide group is interpreted.
\end{abstract}

In der Literatur sind verschiedene Verfahren zur Synthese von Acetessigsäureamiden beschrieben ${ }^{1-6}$. Wohl den elegantesten Weg zur Darstellung dieser Verbindungen stellt die Umsetzung von Diketen mit primären und sekundären Aminen dar. Diese Reak. tion wurde zuerst von $\mathrm{B}$ o e s e beschrieben und hat sich für die Darstellung einer Anzahl aliphatischer und aromatischer Acetessigsäureamide als sehr nützlich erwiesen ${ }^{7-10}$.

Im Rahmen unserer systematischen Untersuchungen über das komplexchemische Verhalten der Acetessigsäureamide stellten wir eine große Anzahl von Uranyl-, Beryllium- und Kupferchelaten mit verschieden substituierten Acetessigsäuredialkylamiden, Acetessigsäureaniliden, -2-amidopyridinen und -2-amidopyrimidinen her und untersuchten die Stabilität der Chelate in Abhängigkeit von den Substituenten der Amidgruppe ${ }^{11-14}$. Als Ergebnis dieser Untersuchungen fanden wir, daß die Stabilität des Chelatringes mit steigender Elektronendichte am Amidstickstoff wächst, und daß somit der induktive Effekt der Substituenten an der Amidgruppe die Stabilität der Metallchelate beeinflußt.

Diese Ergebnisse führten zu der Überlegung, daß Veränderungen an einem der unmittelbar am Chelatring beteiligten Atome eine noch stärkere Beeinflussung der Stabilität der Metallchelate zufolge habe. Aus diesem Grund substituierten wir ein

Sonderdruckanforderungen an Dr. A. KetTrup, RuhrUniv. Bochum, Lehrstuhl f. Anorgan. Chemie I, D-4630 Bochum-Querenburg, Postfach 2148.

1 G. E. Utzinger, Helv. chim. Acta 35, 1359 [1952].

2 L. Wolf u. K. Wetzel, Chem. Ber. 90, 1007 [1957].

3 W. A. Sexton, J. chem. Soc. [London] 1942, 303.

${ }^{4}$ H. E. Fierz-David u. E. Ziegler, Helv. chim. Acta 11, 779 [1928].

5 J. W. Williams u. J. A. Krynitzky, Org. Syntheses, vol. III, p. 10, 1955.

${ }^{6}$ C. D. Hurd u. C. D. Kelso, J. Amer. chem. Soc. 62, 1548 [1940].

7 A. B. Boese, U.S.P. 2.152 .132 (23. 3. 1939).
Wasserstoffatom der mittelständigen $\mathrm{CH}_{2}$-Gruppe durch ein Bromatom und stellten eine Reihe bisher nicht beschriebener substituierter Acetessigsäureamide und ihre Kupferchelate dar, deren Stabilität ermittelt wurde.

\section{Durchführung der Experimente}

\section{Darstellung der 2-Bromacetessigsäureamide}

Die Bromierung der Acetessigsäureamide erfolgte nach einer allgemeinen Arbeitsvorschrift mit $N$-Bromsuccinimid ${ }^{15}$. Die Umsetzung mit Brom/Eisessig nach M e h t a und Mitarbb. ${ }^{16}$ erwies sich bei der Synthese der 2-Bromacetessigsäure-Amide als weniger geeignet, da die Ausbeute und die Reinheit der Produkte unbefriedigend waren.

Die Darstellung der Chelatbildner läßt sich am Beispiel des 2-Bromacetessigsäureanilids wie folgt formulieren:<smiles>CC(=O)C(Br)C(=O)[Y]C(=O)C(Br)C(C)=O</smiles>

8 A. B. Boese, U.S.P. 2.152.786-7 (4. 4. 1939).

9 A. B. Boese, Ind. Engng. Chem. 32, 16 [1940].

10 R. L. Pelley, U.S.P. 3.304.328 [1967].

11 A. Kettrup u. H. Specker, Z. analyt. Chem. 246, 108 [1969].

12 A. Kettrup, Z. analyt. Chem. 246, 183 [1969].

13 G. Stückelmann, A. Kettrup u. H. Specker, Z. anorg. allg. Chem. 372, 134 [1970].

14 G. Stückelmann, A. Kettrup u. H. Specker, Z. anorg. allg. Chem. 372, 144 [1970].

15 L. Horner u. E. H. Winkelmann, Angew. Chem. 79, 349 [1959].

16 C. M. Mehta, J. M. Trivedi u. G. H. Patel, J. sci. Industr. Res. (India) 20 B, 460 [1961]. 
$\mathrm{Zu}$ einer Lösung von $0,1 \mathrm{Mol}$ des betreffenden Acetessigsäureamids in $100-300 \mathrm{ml} \mathrm{CCl}_{4}$ gibt man bei Raumtemperatur $0,1 \mathrm{Mol} N$-Bromsuccinimid und 0,5 mMol $\alpha, \alpha^{\prime}$-Azo-iso-butyronitril als Aktivator. Das $N$. Bromsuccinimid befindet sich zunächst am Boden des Kolbens. Die Reaktionsmischung wird im Wasserbad 45 Min. zum Sieden erhitzt. Die Reaktion ist beendet, wenn die eingesetzte Menge des $N$-Bromsuccinimid als Succinimid auf der Reaktionslösung schwimmt. Man saugt die erkaltete Mischung ab, verwirft den Niederschlag, und zieht am Rotationsverdampfer das Lösungsmittel von dem Filtrat ab. Das Reaktionsprodukt wird in wenig Äthanol umkristallisiert.

\section{Darstellung der Kupferchelate}

Die Darstellung der Kupferchelate erfolgte nicht nach dem üblichen Verfahren in wäßriger Lösung unter Zugabe von Natronlauge, sondern nach einem Verfahren, das schon bei der Synthese von Acetylacetonaten gute Ergebnisse lieferte ${ }^{17}$.

Nach diesem Verfahren löst man 0,01 Mol Chelatbildner in $200 \mathrm{ml}$ Äther und tropft zu der siedenden Lösung $5 \mathrm{mMol}$ Kupferoctoat (Kupfersalz der 2-Äthylcapronsäure) in $50 \mathrm{ml}$ Äther.

Die Reaktion ist nach 30 Min. Erhitzen am Rückfluß abgeschlossen. Der gebildete Niederschlag wird abgenutscht und anschließend am Soxhlet-Apparat mit $70 \mathrm{ml}$ Äther eine Stde. lang extrahiert. Die Kupferchelate wurden durch Elementaranalysen identifiziert. Es handelt sich um 1:2-Chelate.

\section{Potentiometrische Messungen}

Die potentiometrischen Titrationen wurden mit einem Superpotentiographen E 436 der Fa. Metrohm in einem thermostatierten Glasgefäß $\left(20 \pm 0,5^{\circ} \mathrm{C}\right)$ durchgeführt. Als Meßkette diente eine Einstabglaselektrode E 121 UX der gleichen Firma $\left(0-14 \mathrm{pH}, 15-100^{\circ} \mathrm{C}\right)$. Die Elektrode enthielt eine gesättigte $\mathrm{NaCl}$-Lösung. Die Genauigkeit der zur Eichung verwendeten standardisierten Pufferlösungen betrug für die pH-Werte $1-13 \pm$ $0,02 \mathrm{pH}$-Einheiten. Zur Titration wurden $1 \mathrm{mMol} \mathrm{Cu}$ $\left(\mathrm{ClO}_{4}\right)_{2}, 4 \mathrm{mMol}$ Chelatbildner, $1 \mathrm{mMol} \mathrm{HClO}_{4}$ sowie $12,5 \mathrm{mMol} \mathrm{NaClO}_{4}$ in $100 \mathrm{ml}$ eines Dioxan-Wassergemisches $(70 \%$ Dioxan) vorgelegt. Die zur Titration verwendete carbonatfreie $1 \mathrm{~N}$ Natronlauge floß mit einer Geschwindigkeit von $1 \mathrm{ml} / 5 \mathrm{Min}$. zu.

\section{Ergebnisse und Diskussion}

Die von uns dargestellten 2-BromacetessigsäureAmide sowie einige der Kupferchelate sind in Tab. 1 zusammengefaßt.

Die Auswertung der potentiometrischen Titrationen sowie die Berechnung der Stabilitätskonstan-

17 G. Stöckelmann, A. Kettrup u. H. Specker, Angew. Chem. 79, 530 [1967].

18 J. Bjerrum u. P. Andersen, Kgl. dansk. Vidensk. Selsk. 22, 1 [1945]. ten erfolgte in der bereits häufig beschriebenen Weise nach BJERrum ${ }^{18-20}$.

Die ermittelten Dissoziationskonstanten der Chelatbildner sowie die Stabilitätskonstanten der Kupferchelate sind in Tab. 2 aufgeführt.

Wie aus Tab. 2 ersichtlich ist, nimmt die Stabilität der Kupferchelate bei gleichen Substituenten am Phenylring von der $o$ - über die $m$ - bis zur $p$-Stellung hin zu. Bei gleicher Stellung verschiedener Substituenten am Phenylring bilden 2-Bromacetessigsäureanisidide stabilere Metallchelate als die entsprechenden -toluidide und -chloranilide. Unter Beteiligung des aromatischen Systems kann wahrscheinlich das gesamte Chelatbildnermolekül zu einer durchgehenden Konjugation befähigt werden, wobei sich mehrere mesomere Grenzformen überlagern. Substituenten am Phenylring, die einen positiven mesomeren Effekt bewirken, wie z. B. die Methoxyl- oder Methylgruppe erhöhen die Stabilität der Kupferchelate. In diesen Fällen muß die Elektronendichte am Amidstickstoff größer sein als beim Kupferchelat mit 2-Bromacetessigsäureanilid. Als $\mathrm{Ma}$ für die Elektronendichte am Amidstickstoff kann die Basizität des freien Anilins angesehen werden. Trägt man die Stabilitätskonstanten der Kupferchelate gegen die Basizitätskonstanten der freien Aniline auf, so erhält man im Fall der paraund metasubstituierten 2-Bromacetessigsäure-Anilide eine lineare Abhängigkeit, während für die orthosubstituierten Vertreter keine Gesetzmäßigkeit festzustellen ist, wie aus Abb. 1 hervorgeht.

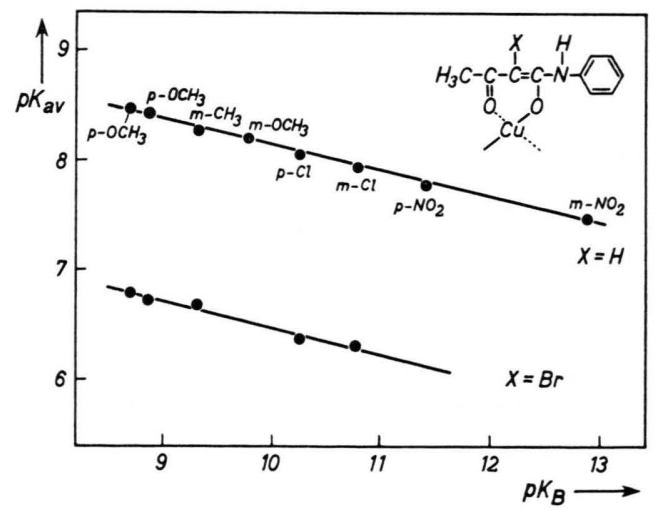

Abb. 1. Beziehung zwischen den Stabilitätskonstanten der Kupferchelate und den Basizitätskonstanten $\mathrm{pK}_{\mathrm{B}}$ der Chelatbildner.

19 M. Calvin u. K. W. Wilson, J. Amer. chem. Soc. 67, 2003 [1945].

20 G. van Uitert, W. C. Fernelius u. B. E. Douglas, J. Amer. chem. Soc. 75, 2736 [1953]. 


\begin{tabular}{|c|c|c|c|c|c|c|c|}
\hline \multirow[t]{2}{*}{ 2-Bromacetessigsäure- } & \multirow[t]{2}{*}{$\begin{array}{l}\text { Schmp. } \\
{\left[{ }^{\circ} \mathrm{C}\right]}\end{array}$} & \multirow[t]{2}{*}{$\begin{array}{c}\text { Ausb. } \\
{[\%]}\end{array}$} & \multicolumn{4}{|c|}{$\begin{array}{c}\text { Analyse } \\
{[\%]}\end{array}$} & \\
\hline & & & & & $\mathrm{H}$ & $\mathrm{N}$ & $\mathrm{Br}$ \\
\hline \multirow[t]{2}{*}{ anilid } & $132-133$ & 64 & ber. & 46,89 & 3,93 & 5,46 & 31,20 \\
\hline & & & gef. & 46,84 & 3,96 & 5,62 & 31,10 \\
\hline \multirow[t]{2}{*}{$o$-toluidid } & $110-112$ & 78 & & 48,90 & 4,47 & 5,18 & 29,58 \\
\hline & & & & 48,79 & 4,56 & 5,19 & 29,55 \\
\hline \multirow[t]{2}{*}{$m$-toluidid } & $81,5-83,5$ & 74 & & 48,90 & 4,47 & 5,18 & 29,58 \\
\hline & & & & 48,74 & 4,48 & 5,05 & 29,45 \\
\hline \multirow[t]{2}{*}{$p$-toluidid } & $102-103,5$ & 53 & & 48,90 & 4,47 & 5,18 & 29,58 \\
\hline & & & & 48,71 & 4,30 & 5,19 & 29,52 \\
\hline \multirow[t]{2}{*}{$o$-anisidid } & $101,5-102,5$ & 89 & & 46,17 & 4,22 & 4,87 & 27,92 \\
\hline & & & & 46,33 & 4,25 & 4,95 & 27,77 \\
\hline \multirow[t]{2}{*}{$p$-anisidid } & $111-112$ & 47 & & 46,17 & 4,22 & 4,87 & 27,92 \\
\hline & & & & 46,45 & 3,97 & 4,93 & 28,13 \\
\hline \multirow[t]{2}{*}{$o$-chloranilid } & $86-87,5$ & 71 & & 41,33 & 3,12 & 4,82 & 27,50 \\
\hline & & & & 42,04 & 3,62 & 4,86 & 26,98 \\
\hline \multirow[t]{2}{*}{$m$-chloranilid } & $91,5-92,5$ & 33 & & 41,33 & 3,12 & 4,82 & 27,50 \\
\hline & & & & 41,40 & 3,20 & 4,77 & 27,24 \\
\hline \multirow[t]{2}{*}{$p$-chloranilid } & $122-123$ & 64 & & 41,33 & 3,12 & 4,82 & 27,50 \\
\hline & & & & 41,39 & 2,89 & 4,75 & 27,54 \\
\hline \multirow[t]{2}{*}{ 2.4-dimethylanilid } & $122-123,5$ & 72 & & 50,72 & 4,96 & 4,92 & 28,12 \\
\hline & & & & 50,85 & 5,06 & 4,93 & 28,29 \\
\hline \multirow[t]{2}{*}{ 3.5-dimethylanilid } & $121-123$ & 89 & & 50,72 & 4,96 & 4,92 & 28,12 \\
\hline & & & & 50,67 & 5,23 & 4,96 & 27,99 \\
\hline \multirow{2}{*}{ 2-methyl-5-chloranilid } & $123,5-124,5$ & 64 & & 43,37 & 3,64 & 4,59 & 26,23 \\
\hline & & & & 43,10 & 3,74 & 4,24 & 26,20 \\
\hline \multirow[t]{2}{*}{ 2-methyl-4-chloranilid } & $128-130$ & 56 & & 43,37 & 3,64 & 4,59 & 26,23 \\
\hline & 20 & & & 43,36 & 3,92 & 4,63 & 26,25 \\
\hline \multirow[t]{2}{*}{ 2.5-dichloranilid } & $117,5-119$ & 87 & & 36,95 & 2,48 & 4,30 & 24,58 \\
\hline & & & & 36,81 & 2,83 & 4,32 & 24,50 \\
\hline$\alpha$-naphthylamid & $140-141$ & 60 & & 54,92 & 3,95 & 4,57 & 26,10 \\
\hline & & & & 55,06 & 4,09 & 4,76 & 26,15 \\
\hline 2.4-dimethoxyanilid & $73-75$ & 45 & & 45,58 & 4,46 & 4,43 & 25,27 \\
\hline & & & & 45,43 & 4,58 & 4,55 & 25,58 \\
\hline 2.5-dimethoxyanilid & $74-76$ & 67 & & 45,58 & 4,46 & 4,43 & 25,27 \\
\hline & & & & 45,72 & 4,75 & 4,60 & 25,47 \\
\hline$p$-äthoxyanilid & $112,5-113,5$ & 31 & & 48,01 & 4,70 & 4,66 & 26,02 \\
\hline & & & & 48,08 & 4,76 & 4,62 & 26,73 \\
\hline benzylamid & $77-80$ & 36 & & 48,90 & 4,47 & 5,18 & 29,58 \\
\hline & & & & 48,95 & 4,44 & 5,25 & 29,70 \\
\hline $\begin{array}{l}\text { Kupfer-bis-2-bromacetessigsäure- } \\
o \text {-anisidid }\end{array}$ & $134-137$ & 71 & & 41.56 & 3,80 & 4,42 & \\
\hline & $10 x-10$ & 11 & & 41,45 & $\begin{array}{l}5,00 \\
3,76\end{array}$ & $\begin{array}{l}4,+2 \\
4,05\end{array}$ & \\
\hline$m$-chloranilid & $141-146$ & 80 & & 37,26 & 2,81 & 4,36 & \\
\hline & & & & 37,53 & 2,51 & 4,39 & \\
\hline$p$-chloranilid & $156-159$ & 61 & & 37,26 & 2,81 & 4,36 & \\
\hline & & & & 37,42 & 2,71 & 4,28 & \\
\hline 2-methyl-5-chloranilid & $140-145$ & 81 & & 39,28 & 3,92 & 4,18 & \\
\hline & & & & 39,64 & 3,96 & 4,12 & \\
\hline$\alpha$-naphthylamid & $135-136$ & 53 & & 49,76 & 3,57 & 4,16 & \\
\hline & & & & 49,92 & 3,62 & 4,12 & \\
\hline$p$-äthoxyanilid & $140-146$ & 62 & & 43,42 & 4,25 & 4,21 & \\
\hline & & & & 43,44 & 4,08 & 4,29 & \\
\hline
\end{tabular}

Tab. 1. 2-Bromacetessigsäureanilide.

In diesen Fällen muß neben dem mesomeren Effekt die sterische Hinderung durch diesen Substituenten einen stabilitätserniedrigenden Einfluß ausüben. Eine Beeinflussung von Chelatring- und Amidresonanz bei den Metallchelaten mit substituierten Aniliden wird unserer Meinung nach da- durch belegt, daß die Stabilitätskonstanten der bisher untersuchten Metallchelate mit Acetessigsäuredialkylamiden höhere Werte besitzt ${ }^{11-13}$.

Die Substitution eines Wasserstoffs der mittelständigen $\mathrm{CH}_{2}$-Gruppe durch ein Bromatom führt zur Störung der Chelatringresonanz und damit zu 


\begin{tabular}{|c|c|c|c|c|}
\hline 2-Bromacetessigsäure- & $\mathrm{pK}_{\mathrm{D}}$ & $\mathrm{pK}_{1}$ & $\mathrm{pK}_{\mathrm{x} v}$ & $\mathrm{pK}_{2}$ \\
\hline anilid & 9,06 & 6,79 & 6,20 & 5,76 \\
\hline$o$-anisidid & 8,97 & 7,20 & 6,55 & 5,96 \\
\hline$p$-anisidid & 9,55 & 7,41 & 6,80 & 6,40 \\
\hline$o$-toluidid & 8,72 & 6,83 & 6,07 & 5,41 \\
\hline$m$-toluidid & 9,55 & 7,25 & 6,68 & 6,30 \\
\hline$p$-toluidid & 9,43 & 7,35 & 6,74 & 6,40 \\
\hline$o$-chloranilid & 8,05 & 6,53 & 5,97 & 5,41 \\
\hline$m$-chloranilid & 8,95 & 6,87 & 6,30 & 5,93 \\
\hline$p$-chloranilid & 8,86 & 6,94 & 6,38 & 6,05 \\
\hline$p$-äthoxyanilid & 9,70 & 7,39 & 6,89 & 6,53 \\
\hline 2.4-dimethoxyanilid & 9,60 & 8,01 & 7,35 & 6,74 \\
\hline 2.4-dimethylanilid & 9,00 & 7,06 & 6,31 & 5,56 \\
\hline 3.5-dimethylanilid & 9,00 & 7,11 & 6,32 & 5,62 \\
\hline 2-methyl-5-chloranilid & 8,30 & 6,54 & 6,18 & 5,82 \\
\hline 2-methyl-3-chloranilid & 8,73 & 7,04 & 6,34 & 5,71 \\
\hline benzylamid & 9,81 & 7,62 & 6,86 & 6,36 \\
\hline$\alpha$-naphthylamid & 8,60 & 7,28 & 6,50 & 5,87 \\
\hline
\end{tabular}

Tab. 2. Dissoziationskonstanten der 2-Bromacetessigsäureamide und Stabilitätskonstanten ihrer Kupferchelate.

einer Stabilitätsverminderung der entsprechenden Metallchelate um ungefähr 1,5 Zehnerpotenzen, wie die Abb. 2 zeigt.

Die Stabilitätsmessungen an Kupferchelaten mit disubstituierten 2-Bromacetessigsäureaniliden stützen die soeben gemachten Aussagen.

Der stabilitätserniedrigende Einfluß des Bromatoms kann sowohl infolge induktiven als auch mesomeren Effekts hervorgerufen werden. Eine Klärung erhoffen wir durch Stabilitätsmessungen an

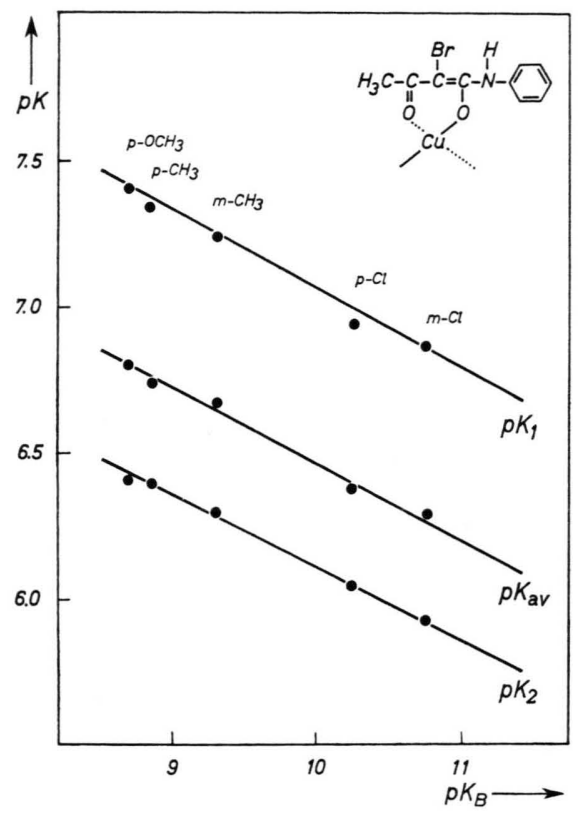

Abb. 2. Auftragung der Stabilitätskonstanten der Kupferchelate mit Acetessigsäureamiden und 2-Bromacetessigsäureamiden gegen die Basizitätskonstanten der freien Aniline.

Kupferchelaten mit 2-Chlor- und 2-Jodacetessigsäureamiden zu erhalten.

Fräulein S. Altreld danken wir für experimentelle Mitarbeit bei den Stabilitätsmessungen. 